\title{
Mice share yeast's ageing system
}

A protein that regulates lifespan in yeast by protecting its genome may perform the same function in mammals, studies in mice suggest. The work addresses a lingering uncertainty about sirtuins, a family of proteins that are a focus of research in the lucrative market for anti-ageing drugs. In April, Sirtris Pharmaceuticals, based in Cambridge, Massachusetts, and co-founded by David Sinclair of Harvard Medical School, who is the lead author of the new work, was purchased by pharmaceutical giant GlaxoSmithKline for US\$720 million.

Researchers already knew that a yeast sirtuin called Sir2 is a sentinel of the organism's genome, preventing genes from being expressed at the wrong times and blocking the chromosomal rearrangements that

sometimes occur in areas of repetitive DNA sequence. But when DNA strands break, Sir2 molecules move to repair the damage, leaving their usual positions unguarded. As cells age, the rate of DNA damage increases, forcing Sir2 proteins to leave their original posts more frequently. Some genes that were meant to be silenced are then free to be expressed, generat-

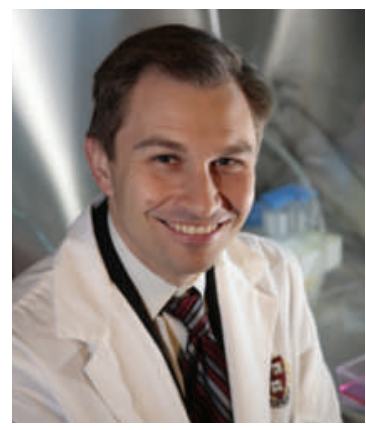

David Sinclair wants to reverse the effects of ageing. ing a shift in patterns of gene expression that is characteristic of ageing.

Increasing Sir2 levels slows ageing in yeast, but it has been unclear whether sirtuins would act by a similar mechanism in mammals. "People, including myself, struggled with the acceptance that a mechanism of ageing in a yeast could be relevant to a human," says Sinclair.

Now, he and his colleagues report that a mouse sirtuin called SIRT1 behaves much like its yeast counterpart (P. Oberdoerffer et al. Cell doi:10.1016/j. cell.2008.10.025; 2008). In mouse embryonic stem cells, SIRT1 also associates with regions of repetitive DNA, and silences the expression of certain genes. But when the cells are treated with hydrogen peroxide, a chemical that can cause DNA damage, SIRT1 is recruited to the site of DNA breakage, and previously silenced genes become expressed. Furthermore, the team found similar changes in geneexpression patterns in brains of elderly mice. "This could be the key to understanding how sirtuins could be beneficial during ageing," says Jan Vijg of the Albert Einstein College of Medicine in New York.

Sinclair now aims to find out whether increasing SIRT1 expression in old mice can revert gene expression to a more youthful pattern - effectively reversing the effects of ageing.

Heidi Ledford 